\title{
Carrier dynamics in submonolayer InGaAs/GaAs quantum dots
}

\author{
Xu, Zhangcheng; Zhang, Yating; Hvam, Jørn Märcher; Xu, Jingjun; Chen, Xiaoshuang; Lu, Wei
}

\section{Published in:}

Applied Physics Letters

Link to article, DOI:

$10.1063 / 1.2219394$

Publication date:

2006

Document Version

Publisher's PDF, also known as Version of record

Link back to DTU Orbit

Citation (APA):

Xu, Z., Zhang, Y., Hvam, J. M., Xu, J., Chen, X., \& Lu, W. (2006). Carrier dynamics in submonolayer InGaAs/GaAs quantum dots. Applied Physics Letters, 89(1), 013113. https://doi.org/10.1063/1.2219394

\section{General rights}

Copyright and moral rights for the publications made accessible in the public portal are retained by the authors and/or other copyright owners and it is a condition of accessing publications that users recognise and abide by the legal requirements associated with these rights.

- Users may download and print one copy of any publication from the public portal for the purpose of private study or research.

- You may not further distribute the material or use it for any profit-making activity or commercial gain

- You may freely distribute the URL identifying the publication in the public portal

If you believe that this document breaches copyright please contact us providing details, and we will remove access to the work immediately and investigate your claim 


\title{
Carrier dynamics in submonolayer InGaAs/GaAs quantum dots
}

\author{
Zhangcheng $\mathrm{Xu}^{\mathrm{a})}$ and Yating Zhang \\ Key Laboratory of Advanced Technique and Fabrication for Weak-Light Nonlinear Photonics Materials \\ (Ministry of Education), TEDA College, Nankai University, Tianjin 300457, People's Republic of China \\ and National Laboratory for Infrared Physics, Chinese Academy of Sciences, Yutian Road No. 500, Shanghai \\ 200083, People's Republic of China \\ Jørn M. Hvam \\ Department of Communications, Optics and Materials, and Nano.DTU, Technical University of Denmark, \\ DK-2800 Lyngby, Denmark \\ Jingjun Xu \\ Key Laboratory of Advanced Technique and Fabrication for Weak-Light Nonlinear Photonics Materials \\ (Ministry of Education), Nankai University, Tianjin 300457, People's Republic of China

\begin{abstract}
Xiaoshuang Chen and Wei Lu
National Laboratory for Infrared Physics, Chinese Academy of Sciences, Yutian Road No. 500, Shanghai 200083, People's Republic of China
\end{abstract}

(Received 4 March 2006; accepted 2 June 2006; published online 6 July 2006)

Carrier dynamics of submonolayer InGaAs/GaAs quantum dots (QDs) were studied by microphotoluminecence (MPL), selectively excited photoluminescence (SEPL), and time-resolved photoluminescence (TRPL). MPL and SEPL show the coexistence of localized and delocalized states, and different local phonon modes. TRPL reveals shorter recombination lifetimes and longer capture times for the QDs with higher emission energy. This suggests that the smallest SML QDs are formed by perfectly vertically correlated two-dimensional InAs islands, having the highest In content and the lowest emission energy, while a slight deviation from the perfectly vertical correlation produces larger QDs with lower In content and higher emission energy. (C) 2006 American Institute of Physics. [DOI: 10.1063/1.2219394]

Self-assembled quantum dots (QDs) can be grown either in the conventional Stranski-Krastanow (SK) mode or via submonolayer (SML) deposition. ${ }^{1-5}$ SML InGaAs/GaAs QD heterostrutures are verified to be a quantum-dot-quantumwell structure (QDQW), in which local clusters with higher In content are embedded in a lateral quantum well with lower In content. ${ }^{6,7}$ Although SML InGaAs QD lasers with high gain or power have been realized recently, ${ }^{5,8,9}$ very few works have been carried out on the carrier dynamics of SML QDs, as compared with SK QDs. ${ }^{1,10-12}$ The study of the carrier dynamics of SML QD structures is of great interest not only for the understanding of the fundamental physics of zero-dimensional structures but also for optoelectronic device applications.

In this letter, we use microphotoluminescence (MPL), selectively excited photoluminescence (SEPL), and time resolved photoluminescence (TRPL) to explore the localization, capture, and recombination of carriers in SML QD structures, at low temperature. We found that the capture time and the recombination lifetime of SML QDs depend strongly on the emission energy, which could be explained by analyzing the growth mechanism of SML QDs.

SML InGaAs/GaAs QDs was formed by alternate deposition of 0.5 ML InAs and 2.5 ML GaAs for ten times (see Ref. 6 for the details of the sample preparation). The MPL and SEPL were measured at $10 \mathrm{~K}$, and the TRPL measurements were carried out at $5 \mathrm{~K}$. In MPL, the incident light from a He-Ne laser at the wavelength of $632.8 \mathrm{~nm}$ was focused on the sample to a spot of around $2 \mu \mathrm{m}$ in diameter. In

\footnotetext{
${ }^{a)}$ Electronic mail: zcxu@nankai.edu.cn
}

SEPL, a wavelength-tunable Ti:sapphire laser was used for excitation. In the TRPL setup, the sample was cooled in a liquid helium cryostat and excited in the GaAs barriers with $120 \mathrm{fs}$ pulses from a Ti:sapphire laser at the wavelength of $800 \mathrm{~nm}$ and the PL signal was collected, dispersed, and synchronously detected using a streak camera with 2.5 ps time resolution. The excitation spots in both REPL and TRPL are about $50 \mu \mathrm{m}$ in diameter.

The size distribution of QD ensembles could vary slightly with the position on the wafer as seen in Fig. 1(a) at low excitation density. The fine structures for the three spectra are different from each other showing emission from individual QDs. These features are reproducible so we could rule out the effect of noise. As the areal density of the SML

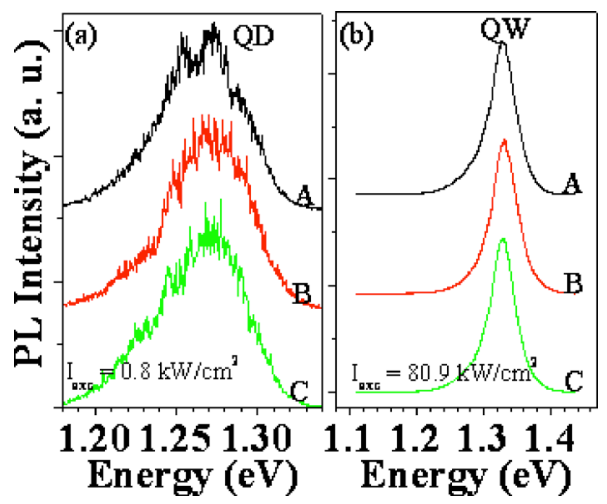

FIG. 1. (Color online) Microphotoluminecence spectra at $10 \mathrm{~K}$ at three different points on the wafer (A, B, and C) $1 \mathrm{~mm}$ apart, at low excitation power density (a) and at high excitation power density (b). 


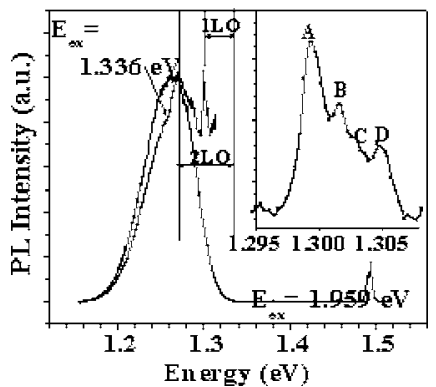

FIG. 2. (Color online) The PL spectra of the SML QD structure at the excitation energies of $1.959 \mathrm{eV}$ above the GaAs barrier band gap and $1.336 \mathrm{eV}$ just above the edge energy of the QW at $10 \mathrm{~K}$. The inset zooms in the $1 \mathrm{LO}$ parts, and the solid line is a guide for the eye.

QDs is about $5 \times 10^{11} \mathrm{~cm}^{-2}$ as reported in Ref. 6, about 15000 SML QDs are probed at the same time. This explains the high density of sharp lines throughout the whole contour of the QD PL emission. At high excitation power density, a peak at $1.326 \mathrm{eV}$ dominates the whole spectrum, and the peak energies for the three excitation points are identical, as shown in Fig. 1(b). This indicates that the peak at $1.326 \mathrm{eV}$ originates from the delocalized states in the studied structure, corresponding to the QW states. ${ }^{7}$

In SEPL measurement with the excitation energy $E_{\mathrm{ex}}$ tuned near to the edge of the density of states (DOS) in the QW $\left(E_{\mathrm{ex}}=1.336 \mathrm{eV}\right)$, a few sharp resonant lines and a resonant PL band appear within the broad PL band, near to one longitudinal optical [(LO) 31-36 meV] and 2LO (66 meV) phonon energies below the excitation energy, respectively, as shown in Fig. 2. To confirm that these sharp lines are not attributed to resonant Raman scattering, the polarization directions of the incident laser beam and the detected PL signal were set to be along the $[110]$ and the $[1, \overline{1}, 0]$ directions, respectively, in the backscattering geometry, as in Ref. 13. A Raman signal cannot be detected in this geometry, according to the selection rules. ${ }^{14}$ When the excitation energy is less than one GaAs LO-phonon energy above the lateral QW ground state in the SML-grown QDQW structure, the probability for the photon-excited carriers (excitons) to relax within the QW states by emission of only longitudinal acoustic (LA) phonons is less than the carrier (exciton) capture probability from QW to QDs by emission of LO phonons. Dots which can be accessed by emission of LO phonons are populated more efficiently, since their delta-function-like DOS can be accessed directly from the excited energy level in the QW by LO phonon emission.

The complex structure in the $1 \mathrm{LO}$ resonant peaks consists of several optical phonon modes whose energies are $36.7,34.5,32.9$, and $31.3 \mathrm{meV}$, respectively, as shown in the inset of Fig. 2. We assign these lines to the LO phonon modes in the GaAs barrier, the GaAs/InAs interface, the InGaAs lateral QW, and the InGaAs QDs, respectively. The $2 \mathrm{LO}$ resonance occurs at the energy of $66 \mathrm{meV}$ below the excitation energy, nearly two times the LO phonon energies of QW. The coexistence of several optical phonon modes indicates the complex structure of SML QDs.

The electron-hole $(e-h)$ pairs (or excitons) generated in the GaAs barrier are either captured directly into the QW where they relax and are finally captured by the QDs, or they are directly captured by the QDs or recombine in the QW. Then the captured carries will recombine inside the QDs.
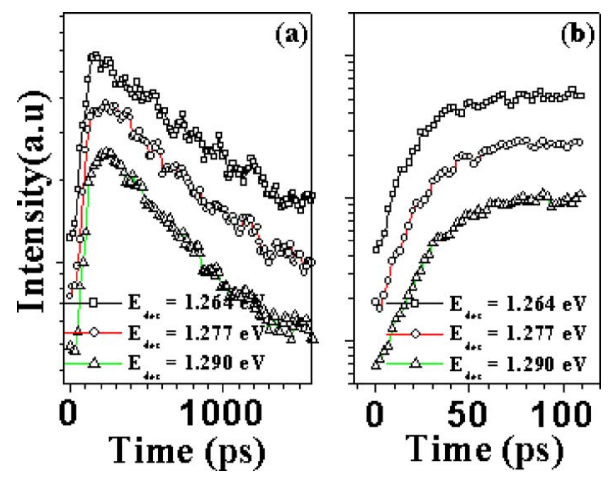

FIG. 3. (Color online) TRPL detected at different ground states of SML QDs at $5 \mathrm{~K}$, (a) in the long time scale for the evaluation of the decay time; (b) in the short time scale for the evaluation of the rise time.

Figure 3 shows the PL transient of QD states in SML InGaAs/GaAs QD structures, at an excitation density of $101 \mathrm{~W} / \mathrm{cm}^{2}$ (corresponding to $10^{17}$ electron-hole pairs/ $\mathrm{cm}^{3}$ pulse). On the long timescale [Fig. 3(a)], the PL decay can be well fitted by a monoexponential function, and the decay time $\tau_{d}$ can be evaluated. On the short timescale [Fig. $3(\mathrm{~b})]$, the PL transients can be fitted by the expression ${ }^{15}$

$$
I(t) \propto\left[\exp \left(-t / \tau_{r}\right)-\exp \left(-t / \tau_{d}\right)\right] /\left(\tau_{r}-\tau_{d}\right),
$$

where $\tau_{r}$ is the rise time of PL transients, which can provide information on carrier capture into the QDs.

Fig. 4 shows the values of $\tau_{d}$ and $\tau_{r}$ plotted against the QD emission energy. With increasing QD transition energy,

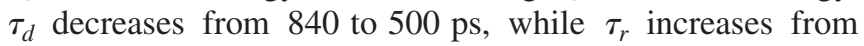
35 to $60 \mathrm{ps}$

For SK QDs, QDs with higher emission energy are believed to be smaller, and stronger electron-hole overlap occurs inside the QDs, resulting in longer lifetime. ${ }^{16}$ Recently, a reduction of the radiative lifetime for smaller SK QDs with higher emission energy has been observed, which was explained by the reduced electron-hole overlap integral due to the larger piezoelectric effect in larger QDs. ${ }^{17}$ However, in

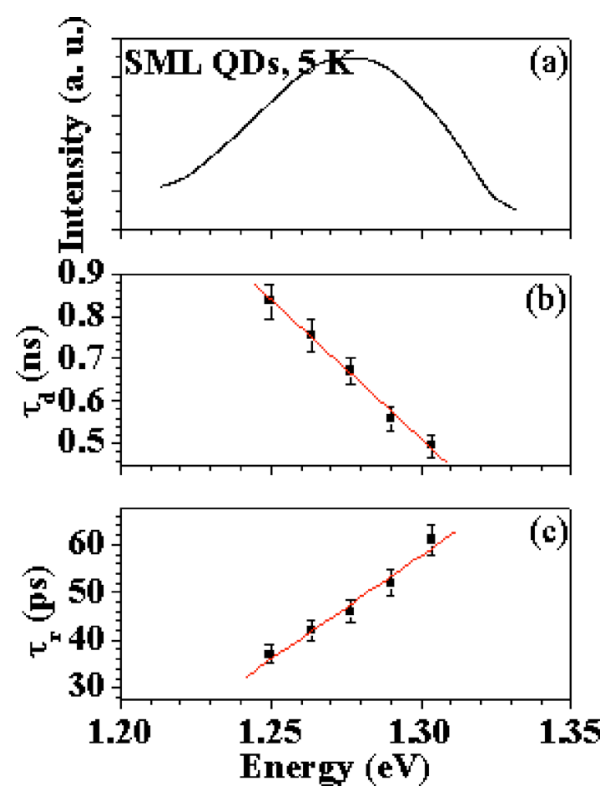

FIG. 4. (Color online) Dependence of the decay (rise) time with respect to the QD emission energy for SML QDs. (a) the integrated PL spectra; (b) the decay time; and (c) the rise time.

AlP license or copyright; see http://apl.aip.org/apl/copyright.jsp 


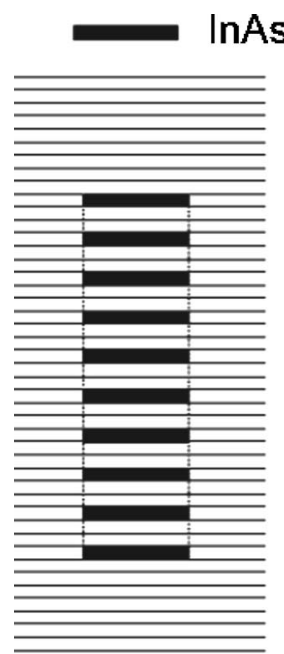

SQD

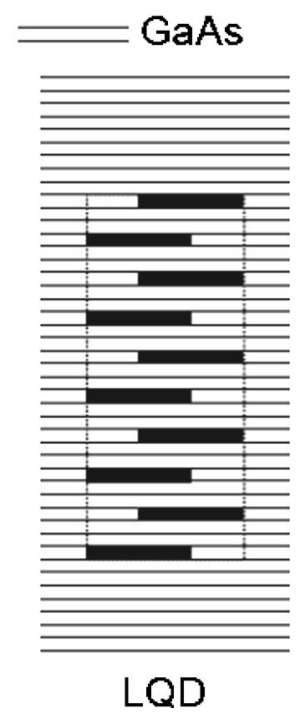

FIG. 5. (Color online) The schematic diagram showing the relationship between the size and average In content inside SML QDs. The QD regions are circled by the dotted lines. SQD and LQD are referred to as small QDs and larger QDs.

the case of SML QDs, the smallest QDs are formed by perfectly vertically correlated two-dimensional (2D) InAs islands, ${ }^{2,3,6,8}$ and have the highest In content, while slight deviation from the perfect vertical correlation produces larger QDs with lower In content, as schematically shown in Fig. 5. SML QDs with higher emission energy have larger lateral dimensions but the same height, as suggested by the in-plane transmisson electron microscope (TEM) image in Ref. 6. The contrast of the image comes from the difference of In contents, the brighter parts have less In contents than the darker parts. Although it is not easy to distinguish the difference of In contents between the smaller and larger QDs, we can clearly see the contrast difference between the lateral InGaAs QWs (bright parts) with lower In contents and the QDs (darker papers) with higher In content. The lateral InGaAs QW structure is formed due to the random distribution of InAs 2D islands within the GaAs matrix. In some sense, the QW structure can be regarded as the largest QDs with the lowest In content. The observed shorter PL decay time for larger SML QDs with higher emission energy may thus be due to the enhanced overlap of the electron-hole wave functions. On the other hand, it is also possible that the exciton wave function extension increases, due to the coupling to QW states, with the increase of the QD emission energy, which will make the PL decay time even shorter.

Since thermalization and relaxation processes with the three-dimensional GaAs and the two dimensional QW occur on a much faster time scale, ${ }^{18}$ the measured rise time mainly reflects the capture process into the QD. When the density of carriers generated by each pulse is much higher than the QD density as in the present case, the carrier capture is mediated by Coulomb scattering (Auger process). According to Ref. 19, the Auger coefficient (capture time) decreases (increases) with the increase of the QD diameter, which coincides with the present result.

In summary, we have explored the carrier dynamics of an InGaAs/GaAs QDQW structure formed by submonolayer deposition. The coexistence of the localized states of QDs and the delocalized states of QWs are revealed clearly in the MPL spectra. Different local phonon energies of the QDQW structure are obtained when the excitation energy is tuned close to the edge of DOS of QWs, indicating the complex structure of SML QDs. The recombination lifetime of SML QDs decreases with the increase of QD emission energy. This can be explained by assuming that SML QDs with higher emission energy have lower average In content and larger volume. The Auger carrier capture time for SML QDs increases with the increase of QD volume, which coincides with theoretical predictions. ${ }^{19}$

Enlightening discussions with Vadim Lyssenko and Dan Birkedal are gratefully acknowledged. This work has been supported in part by the National Natural Science Foundation of China (Grant Nos. 60444010, and 60506013), the Danish Technical Science Research Council, the SRF for ROCS (SEM), the Startup fund for new employees of Nankai University, the Marubun Research Promotion Foundation and the PCSIRT.

${ }^{1}$ D. Bimberg, M. Grundmann, and N. N. Ledentsov, Quantum Dot Heterostructures (Wiley, New York, 1999).

${ }^{2}$ V. A. Shchukin and D. Bimberg, Rev. Mod. Phys. 71, 1125 (1999).

${ }^{3}$ V. Bressler-Hill, A. Lorke, S. Varma, P. M. Petroff, K. Pond, and W. H. Weinberg, Phys. Rev. B 50, 8479 (1994).

${ }^{4}$ N. N. Ledentsov, I. L. Krestnikov, M. Strabburg, R. Engelhardt, S. Rodt, R. Heitz, U. W. Pohl, A. Hoffmann, D. Bimberg, A. V. Sakharov, W. V. Lundin, A. S. Usikov, Z. I. Alferov, D. Litvinov, A. Rosenauer, and D. Gerthsen, Thin Solid Films 367, 40 (2000).

${ }^{5}$ A. F. Zhukov, A. R. Kovsh, S. S. Mikhrin, N. A. Maleev, V. M. Ustinov, D. A. Livshits, I. S. Tarasov, D. A. Bedarev, M. V. Maximov, A. F. Tsatsulnikov, I. P. Soshnikov, P. S. Kopev, Z. I. Alferov, N. N. Ledentsov, and D. Bimberg, Electron. Lett. 35, 1845 (1999).

${ }^{6}$ Z. C. Xu, D. Birkedal, J. M. Hvam, Z. Y. Zhao, Y. M. Liu, K. T. Yang, A. Kanjilal, and J. Sadowski, Appl. Phys. Lett. 82, 3859 (2003).

${ }^{7}$ Z. C. Xu, K. Leosson, D. Birkedal, V. Lyssenko, J. M. Hvam, and J. Sadowski, Nanotechnology 14, 1259 (2003).

${ }^{8}$ S. S. Mikhrin, A. E. Zhukov, A. R. Kovsh, N. A. Maleev, V. M. Ustinov, Y. M. Shernyakov, I. P. Soshnikov, D. A. Livshits, I. S. Tarasov, D. A. Bedarev, B. V. Volovik, M. V. Maximov, A. F. Tsatsulnikov, N. N. Ledentsov, P. S. Kopev, D. Bimberg, and Z. I. Alferov, Semicond. Sci. Technol. 15, 1061 (2000).

${ }^{9}$ Z. C. Xu, D. Birkedal, M. Juhl, and J. M. Hvam, Appl. Phys. Lett. 85, 3259 (2004).

${ }^{10}$ Thomas F. Boggess, L. Zhang, D. G. Deppe, D. L. Huffaker, and C. Cao, Appl. Phys. Lett. 78, 276 (2001).

${ }^{11}$ M. De Giorgi, C. Lingk, G. von Plessen, J. Feldmann, S. De Rinaldis, A. Passaseo, M. De Vittorio, R. Cingolani, and M. Lomascolo, Appl. Phys. Lett. 79, 3968 (2001)

${ }^{12}$ L. Ya. Karachinsky, S. Pellegrini, G. S. Buller, A. S. Shkolnik, N. Yu. Gordeev, V. P. Evtikhiev, and V. B. Novikov, Appl. Phys. Lett. 84, 7 (2004).

${ }^{13}$ P. D. Wang, N. N. Ledentsov, and C. M. Sotomayor Torres, J. Appl. Phys. 79, 7166 (1996)

${ }^{14} \mathrm{P}$. Y. Yu and Manuel Cardona, Fundamentals of Semiconductors (Springer, Berlin, 1996).

${ }^{15}$ B. Ohnesorge, M. Albrecht, J. Oshinowo, A. Forchel, and Y. Arakawa, Phys. Rev. B 54, 11532 (1996).

${ }^{16}$ Gustavo A. Narvaez, Gabriel Bester, and Alex Zunger, Phys. Rev. B 72, 245318 (2005)

${ }^{17}$ L. Ya. Karachinsky, S. Pellegrini, G. S. Buller, A. S. Shkolnik, N. Yu. Gordeev, V. P. Evtikhiev, and V. B. Novikov, Appl. Phys. Lett. 84, 7 (2004).

${ }^{18}$ J. Shah, Ultrafast Spectroscopy of Semiconductors and Semiconductor Nanostructures, Solid-State Sciences (Springer-Verlag, Berlin 1999).

${ }^{19}$ I. Magnusdottir, S. Bischoff, A. V. Uskov, and J. Mørk, Phys. Rev. B 67, 205326 (2003). 